\title{
Prävalenz, Erkennens- und Verschreibungsverhalten bei depressiven Syndromen. Eine bundesdeutsche Hausarztstudie
}

\author{
F. Jacobi (1), M. Höfler (1,2), W. Meister (3), H. U.Wittchen (1,2) \\ 1 Klinische Psychologie und Psychotherapie, TU Dresden \\ 2 Max-Planck-Institut für Psychiatrie, München \\ 3 GlaxoSmithKline GmbH \& Co.KG, München
}

\section{Zusammenfassung}

Die Studie berichtet die Stichtags-Prävalenz depressiver Syndrome bei 20.421 unselegierter Patienten, die an einem Stichtag ihren Hausarzt aufsuchten. Zusätzlich wurde das hausärztliche Erkennungs- und Verschreibungsverhalten ermittelt. Im Rahmen einer Vorstudie wurden die teilnehmenden Arztpraxen $(\mathrm{N}=633)$ mittels Fragebogen charakterisiert. Am Erhebungstag erhielten alle Patienten Fragebögen, darunter den „Depression Screening Questionaire“ (DSQ), um die Diagnose einer depressiven Störung gemäß der Kriterien von DSM-IV oder ICD-10 zu stellen. Diese Daten wurden mit den Angaben in den Bewertungsbögen der Ärzte verglichen, die diese am Erhebungstag für jeden Patienten ausfüllten. Die Befunde bestätigen die hohe Prävalenz depressiver Syndrome in der Hausarztpraxis: 11,3\% erfüllten die ICD-10-Kriterien für eine depressive Episode. Obwohl die Erkennungsrate von 59\% wie auch die Häufigkeit verordneter Behandlungen tendenziell höher erscheint als in früheren Studien, so gibt doch die Situation bei den Depressionen leichterer Ausprägung sowie der hohe Anteil an „Fehldiagnosen“ bei Patienten, die deutlich die Falldefinitionskriterien verfehlen, Anlass zur Besorgnis.

\section{Schlüsselwörter}

Depression in der primärärztlichen Versorgung, Diagnostik subklinischer und klinischer Depressionen, Prävalenz, Erkennungsrate

\section{Prevalence, recognition, and prescription behaviour in depressive syndromes. A German study}

\section{Summary}

This study examines the prevalence of depressive syndromes among unselected primary care patients as well as doctors' recognition and treatment rates. This nationwide study included a total of 20,421 patients attending their primary care doctors $(n=633)$ on the study's target day. Patients received a selfreport questionnaire including the depression screening questionnaire (DSQ) to provide diagnoses of depressive disorders according to the criteria of DSM-IV and ICD- 10.These data were compared to the doctor's evaluation form for each patient to assess the physician's diagnostic decision, assessment of clinical severity, and treatment choices. The findings confirm the high prevalence of depressive syndromes in primary care settings $(11.3 \%)$ and underline the particular challenge posed by a high proportion of patients near the diagnosis threshold. Although recognition rates among more severe major depressive patients (59\%) as well as treatments prescribed appear to be more favourable than in previous studies, the situation in less severe cases and the high proportion of doctors' definite depression diagnoses in patients who do not fulfill the diagnostic criteria still raise significant concerns.

\section{Keywords}

Depression in primary care practice, Diagnosis of subclinical and clinical depression, Prevalence, Recognition rate 
Die zunehmend bedeutsame Rolle, die dem Hausarzt nicht nur bei dem Erkennen, sondern auch in der Therapie depressiver Erkrankungen zukommt, ist seit einigen Jahren ein Thema der epidemiologischen und klinischen Public- Health-Forschung. Dabei ergaben Studien in den 80er und frühen 90er Jahren in Deutschland und verschiedenen anderen Ländern bzw. in internationalen Kollaborationen [4, 5, 11, 12, 15, 18, 20, 22, 23], dass depressive Syndrome in der Hausarztpraxis mit Schätzungen von 7-11\% aller Patienten ein überaus häufiges Phänomen sind. Es besteht nach diesen Studien auch ein einigermaßen durchgehender Konsens, dass von den Patienten mit klinisch bedeutsamer Depression zumindest jeder zweite vom Hausarzt nicht als solcher erkannt werden [13, 17, 23, 24]. Unter denen, die als depressiver „Fall““ erkannt wurden, erhält nur ein Bruchteil eine Behandlung, die gemäß fachlicher Leitlinien als adäquat bezeichnet werden kann. Dabei wird speziell im deutschen Sprachraum auf die vergleichsweise hohen Raten von Sedativa-Verschreibungen, sowie die im Vergleich zu trizyklischen Antidepressiva niedrigeren Verschreibungsraten an SSRIs hingewiesen $[2,5,7,16]$.

Die Faktoren, die für diese kritische Situation in der Hausarztpraxis verantwortlich sind, sind immer noch unzureichend untersucht. Bezüglich der Erkennensrate gibt es lediglich Befunde, dass Depressionen mit geringer Symptomanzahl und ohne erhebliche Beeinträchtigungen [17] weniger wahrscheinlich erkannt werden als Depressionen mit größerer Symptombelastung und Beeinträchtigung. Weiter wird vermutet, dass viele depressive Hausarztpatienten sich mit unspezifischen somatischen Beschwerden vorstellen, die die Aufmerksamkeit von einer spezifischen Depressionsdiagnostik ablenken [1, 3, 21].

Darüber hinaus muss darauf hingewiesen werden, dass für Deutschland aktuelle Zahlen weitgehend fehlen. Als Referenz gelten immer noch die Zahlen der bislang vielleicht umfassendsten internationalen WHO-Studie [23] mit den zwei deutschen Zentren Mainz und Berlin [12] aus dem Erhebungsjahr 1991.Da dem Problem unerkannter Depressionen in der primärärztlichen Versorgung in den letzten Jahren zunehmend Beachtung geschenkt wurde, ist von Interesse zu prüfen, inwieweit sich zwischenzeitlich Veränderungen ergeben haben. In der im Folgenden berichteten Studie zur Punktprävalenz depressiver Syndrome wurden an einer bundesweiten Stichprobe von 633 Hausarztpraxen im Jahre 1999 folgende Fragestellungen untersucht:

1. Wie hoch ist die (2-Wochen-)Punkt- Prävalenz depressiver Symptome und Störungen definiert analog ICD-10 und DSM-IV - unter Hausarztpatienten, die ihren Arzt an einem zufällig gewählten Stichtag aufsuchten?

2.Welcher Anteil der Fälle, die die DSM- oder ICD-Kriterien für eine derzeitige depressive Episode erfüllen, werden vom behandelnden Arzt auch als Depression erkannt, und welche Interventionen kommen zum Einsatz?

\section{Methoden}

Diese Studie basiert auf der Untersuchung von N=20.421 unselegierten Patienten, die am Stichtag der Studie (15.April 1999) in den 633 ausgewählten teilnehmenden Hausarztpraxen erschienen waren.

Da aufgrund finanzieller und logistischer Komplikationen eine stratifizierte bundesweite Zufalls-Stichproben-Ziehung aus dem Ärzteregister nicht machbar war (betreuungstechnischer Aufwand, Reisekosten, Zeitaufwand), wurden die Studienärzte unter Zuhilfenahme der zur Verfügung stehenden Arztregister der Firma SmithKline Beecham gezogen. Alle 99 regionalen Repräsentanten der Firma wurde gebeten, etwa 5 „typische“ Hausarztpraxen in ihrer Region zu rekrutieren und als Studienmonitoren der Studie für bis zu 9 schließlich ausgewählten Hausärzte zu fungieren (eingehende Beschreibung s. [26]). Sie wurden organisatorisch unterstützt durch Außendienstmitarbeiter, die jedoch bei der 
konkreten Datensammlung nicht involviert waren. Jegliche Werbung war im Rahmen der Studie untersagt; die teilnehmenden Arztpraxen erhielten für den organisatorischen Mehraufwand eine geringe Aufwandsentschädigung.

Um die Repräsentativität der Arztauswahl zu überprüfen, wurde die Praxen- Stichprobe noch einmal mit statistischen Angaben der Kassenärztlichen Bundesvereinigung [9] verglichen. Es ergaben sich keine signifikanten Unterschiede bezüglich der Verteilung von Allgemeinärzten, praktischen Ärzten und Internisten mit hausärztlicher Funktion sowie der regionalen Verteilung.

Zunächst wurden die teilnehmenden Ärzte 5 Wochen vor dem Stichtag gebeten, einen Vorstudienfragebogen auszufüllen, in dem Informationen über Arbeit und Patientengut, Arztund Praxischarakteristika (Berufserfahrung, Ausbildung und postgraduale Qualifizierungen etc.) sowie die Einstellung gegenüber psychischer Gesundheit und dem Umgang mit Depressionen erfragt wurden. Daraufhin instruierten die regionalen Studienbetreuer die Hausärzte über das Studiendesign, den Studienablauf und die in der Studie verwendeten Instrumente. Außerdem wurde das jeweilige Hilfs- und Pflegepersonal in die logistischen Aspekte der Studie eingewiesen. Nach dieser Trainingsperiode wurden die Patienten, die die Praxen der teilnehmenden Hausärzte am Stichtag aufsuchten, informiert und ihre Bereitschaft zur Teilnahme an der Studie eingeholt (,,informed consent").Außerdem füllten die Patienten den zweiseitigen Patientenfragebogen aus. Die Ärzte wurden gebeten, während oder nach der Konsultation ein Formular zu Diagnostik und Behandlung für jeden Patienten auszufüllen.

\section{Stichprobe der Hausarztpraxen}

52,4\% der Ärzte waren Allgemeinärzte, 20,1\% praktische Ärzte und 27,5\% Internisten mit primärärztlicher Ausrichtung; die mittlere Praxiserfahrung betrug 14 Jahre. Die Mehrheit der Ärzte hatte ein tägliches Patientenaufkommen von mindestens 60 Patienten.

13,6\% hatten zusätzliche Qualifikationen im Bereich Psychotherapie vorzuweisen, und 59\% gaben an, in den letzten 2 Jahren mindestens 3 Weiterbildungskurse zum Thema Depressionen absolviert zu haben. Die Selbsteinschätzungen zur Kompetenz ergaben, dass die Ärzte ihre Fähigkeit, Depressionen zu erkennen und zu diagnostizieren, häufiger als ,gut“ bewerteten (66,6\%) als ihre Kompetenz medikamentöser (48\%) oder psychologischer $(29,6 \%)$ Behandlung.

\section{Untersuchte Patienten}

24.218 Patienten füllten am Stichtag den Patientenfragebogen aus. Für insgesamt 20.421 Patienten lag ein vollständiger Datensatz vor, bei dem sowohl Patientenfragebogen als auch der entsprechende Arztfragebogen zur Verfügung stand. Die Gründe für Unvollständigkeit waren: Teilnahmeverweigerung durch Patient ( $\mathrm{N}=701)$, nur Teile des Fragebogens ausgefüllt ( $\mathrm{N}=844$ ), Identifikations-/Codierungsfelder nicht (richtig) ausgefüllt ( $\mathrm{N}=2252)$. Die folgenden Analysen beruhen auf diesen 20.421 Patienten mit der vollständigen Information.

Gemäß dem Studienprotokoll sollten alle Patienten erreicht werden, die den Arzt am Stichtag aufsuchten. Ausschlusskriterien waren: Alter unter 15 Jahre, schwere kognitive oder wahrnehmungsbedingte Beeinträchtigungen, sowie - aus ethischen Erwägungen - akute schwere Schmerzen und Leiden (z.B. bei Notfallpatienten).Patienten, die an diesem Tag nicht persönlich beim Arzt vorsprachen (z.B. nur Laboruntersuchungen oder Abholen von Rezepten) wurden ebenfalls nicht in die Studie aufgenommen.

Eine Darstellung der soziodemographischen Patientenvariablen findet sich in Tabelle 1. Die am Stichtag ihren Hausarzt aufsuchenden Patienten waren überwiegend Frauen (60\%) höheren Alters (49\% waren älter als 54). Entsprechend der Altersstruktur waren 32,4\% 
berentet. Der Anteil der nichtberufstätigen Hausfrauen lag bei 12,8\%, der der Arbeitslosen bei 8,2\%. Die häufigsten Gründe der Patienten dafür, an diesem Tag den Arzt aufzusuchen, waren somatische Beschwerden und Krankheiten (67,4\%; Mehrfachangaben möglich), gefolgt von Schmerzen (46,0\%) und Schlafproblemen (33,9\%). Psychologische Beschwerden oder Probleme mit der psychischen Gesundheit wurden nur von 8,7\% der Männer $(\mathrm{N}=493)$, jedoch von $13,0 \%$ der Frauen $(\mathrm{N}=1089)$ angegeben $(\mathrm{p}<0,05)$.

\section{Patientenfragebogen und diagnostische Beurteilung}

Im Patientenfragebogen war der Depression Screening Questionnaire (DSQ) [25, 28] enthalten. Der DSQ wurde als zentrales diagnostisches Maß benutzt. Er besteht aus 12 Items, die um 3 Fragen zur Erhebung des Alters zum Zeitpunkt der ersten und der derzeitigen Episode und zur Zahl der Episoden einer bestehenden Depression (2 Wochen, entsprechend der Kriterien von DSM-IV und ICD-10) ergänzt werden. Jedes der Symptom-Items soll auf einer 3-stufigen Skala eingeschätzt werden (liegt an den meisten Tage vor $=2$, an einigen Tagen $=1$, nie $=0$ ). Der DSQ wurde auf der Basis von WHO-CIDI entwickelt, so dass die Items entsprechend der CIDI-Fragen gestaltet sind und den Inhalt von 9 DSM-IV und 10 ICD-10 Depressionskriterien wiedergeben. Entsprechend der Konventionen im DSMIV wird die Diagnose eines ,schweren depressiven Syndroms“ bzw. Major-Depression vergeben, wenn zumindest 5 der entsprechenden Items mit ,liegt an den meisten Tagen vor" beantwortet wurden (Gesamtsumme von 10 oder mehr). Die Kriterien einer milden depressiven Episode gemäß ICD-10 erfordern mindestens 4 Items (darunter 2 depressive Leitsymptome); der Mindestwert im DSQ für Depression nach ICD- 10 muss also mindestens 8 betragen. Die interne Konsistenz des DSQ beträgt 0,83 (Cronbachs $\alpha$ ), die Retestreliabilität reicht von $\kappa=0,66-1,0$. Die Validität der DSQ-Diagnose (verglichen mit der CIDI-Diagnose nach DSMIV und IDC- 10 von depressiven Störungen) beträgt $\kappa=0,89$ (für DSM) und 0,84 (für ICD-10) [25].

\section{Arztfragebogen}

Der Arztfragebogen bestand aus einer Seite mit Fragen zur Einschätzung des Arztes zum allgemeinen klinischen Schweregrad (severity of illness score, CGI) [6], der Einschätzung über die Wahrscheinlichkeit, dass die Person an einer Depression leidet, sowie einer Reihe von Fragen dazu, ob bei dem jeweiligen Patienten vergangene Episoden oder Behandlungen einer Depression bekannt seien und welche Art der Behandlung für diesen Patienten in der derzeitigen Episode gewählt worden ist.

\section{Ergebnisse}

\section{Punktprävalenz depressiver Störungen nach DSM-IV und ICD-10 unter Patienten der} primärärztlichen Versorgung

In Tabelle 2 sind die Prävalenzdaten gemäß der von den Patienten angegebenen Beschwerden nach Geschlecht und mit zugehörigen Vertrauensintervallen aufgeführt.

Entsprechend dem DSQ und dessen diagnostischen Konventionen erfüllten 4,2\% aller primärärztlichen Patienten am Stichtag die Kriterien für eine Major- Depression gemäß DSMIV. Die Wahrscheinlichkeit, die Kriterien nach DSMIV zu erfüllen, war für Frauen höher als für Männer (OR=1,63,95\% ci=1,39-1,90). Die (nicht dargestellte) Überprüfung von möglichen Prädiktoren ergab, dass Probanden über 40 Jahre häufiger betroffen waren als Personen unter $40(\mathrm{OR}=1,22,95 \% \mathrm{ci}=1,04-1,44)$.Ebenso waren die Variablen 
Arbeitslosigkeit, Berentung und Hausfrau verglichen mit Berufstätigkeit Prädiktoren für eine erhöhte Diagnosewahrscheinlichkeit.

Auch wenn die klinische Grenze für die Diagnose bei der IDC-10 nur geringfügig niedriger liegt, ergab sich eine substanziell größere Zahl von Patienten, die die Depressionskriterien nur nach ICD- 10 erfüllten. Dies legt nahe, dass offensichtlich ein hoher Prozentsatz der Patienten die Kriterien für eine „leichtgradige“ depressive Episode nach IDC-10 erfüllten, die lediglich 4 statt der in DSM-V geforderten 5 Symptome erfordert. Die Beziehungen zu Alter, Geschlecht, beruflicher und ehelicher Status waren allerdings für die ICD-10-Fälle beinah identisch zu den DSM-IV-Fällen.

\section{Erkennungsrate}

Tabelle 3 gibt die diagnostischen Entscheidungen der Ärzte wieder und stellt sie den DSMbzw. ICD-Diagnosen auf Grundlage der Patientenangaben gegenüber. Nehmen wir die hausärztliche Diagnose ,sicher und voll ausgeprägt“ als Standard, wurden vom Hausarzt $74,8 \%$ der DSM-Major-Depressionen richtig identifiziert, jedoch nur 49,7\% der ICD-Fälle. Zusätzlich 17,2\% der DSM-Fälle wurden als unterschwellig ausgeprägt erachtet (ICD: 31,5\%). Somit blieben lediglich 8,1\% aller DSM-Major-Depressionen unerkannt oder unklar (ICD: 18,9\%). Die Sensitivität der ärztlichen Urteile kann also als ermutigend hoch bewertet werden.

Auch wenn die Übereinstimmung der ärztlichen Urteile mit den Patientenangaben als Kriterium bezüglich der Fälle, bei denen aufgrund der Patientenangaben definitiv weder eine DSM noch eine ICD-Depression vorlag, mit 60,4\% ebenfalls hoch ist, so bleibt doch die Rate „falscher positiver“ Diagnosen bemerkenswert: $11,7 \%$ der Probanden, die aufgrund der Patientenfragebögen weder DSM noch ICD-Kriterien erfüllten, erhielten eine „sichere und definitive“ Depressionsdiagnose, weitere 28\% eine ,unterschwellige“ Diagnose. Die Spezifität der ärztlichen Urteile ist also deutlich geringer als die Sensitivität.

\section{Interventionen, die laut Arzturteil indiziert sind}

Im Arztbogen wurde erfragt, welche medikamentösen und nichtmedikamentösen Interventionen der Arzt bei von ihm als depressiv diagnostizierten Patienten für indiziert hielt. In Tabelle 4 sind diese Angaben für die DSM-Diagnosen, die nur-ICD-Diagnosen sowie für die „falschen Positiven“ (weder DSM- noch ICD-Depression) aufgeführt.

In den meisten Fällen wird in irgendeiner Form medikamentös behandelt (DSM: 72,7\%, ICD: 60,8\%).Am häufigsten ist die Verschreibung von Antidepressiva: Bei 37,6\% der ärztlichen

Depressionsdiagnosen, die im Sinne der DSM-Kriterien richtig erkannt wurden (ICD: 28,1\%), verschrieb der Arzt „,moderne“ Antidepressiva (SSRI, SNRI, NaSSA etc.) und bei 27,7\% (ICD: 16,2) „klassische“ Antidepressiva (TCA).

Ausschließlicher Einsatz von Beratung/ Krisenintervention sowie Psychotherapie ohne begleitende Medikation nahmen nur den 3. Rangplatz ein (DSM: 11,5\% bzw. 8,3\%; ICD: $18,8 \%$ bzw. 6,1\%).

Keine Intervention erfolgte bei 4,5\% der DSM- und bei 11\% der ICD-Fälle. Zum Spezialisten (psychiatrische, psychotherapeutische oder stationäre Behandlung) wurden 16,2\% der DSMbzw. 10,1\% der ICD-Fälle überwiesen.

Bemerkenswert ist der Befund einer hohen Anzahl von Interventionen bei Patienten, die weder DSM- noch ICD-Kriterien für eine Depression erfüllen, aber vom Arzt als definitiv depressiv diagnostiziert wurden (,falsche positive“ Depressionsfälle). Aus der 3. Spalte der Tabelle 4 ist ersichtlich, dass sich die Interventionen bei diesen Fällen nur unwesentlich von der Behandlung „,echter“ Depressionen unterscheiden. Die unvollständigen „Verbatim“- 
Beschreibungen der Ärzte zu ihren Fällen lassen erkennen, dass vermehrt psychosomatische und Angststörungen hier klassifiziert wurden.

\section{Diskussion}

Es müssen bei der Interpretation der Ergebnisse verschiedene Begrenzungen der Studie berücksichtigt werden.

1. Als Maßstab für eine Diagnose nach den DSM- bzw. ICD-Kriterien wurde aus Effizienzüberlegungen der prototypische Patientenfragebogen DSQ verwendet. Dies ist offensichtlich als Datenbasis weniger differenziert als bewährte klinische Interviews und bildet in erster Linie reliabel das Vorliegen eines depressiven Syndroms ab, ohne die erforderlichen Ausschlusskriterien zu beachten. Allerdings ist die Sensitivität des DSQ als ausgesprochen hoch zu bewerten ( $>95 \%)$. Dies führt aufgrund der psychometrische Voruntersuchungen [25] zu einer Überschätzung von 6-8\% der wahren Depressionsrate, da Depressionen im Rahmen bipolarer Erkrankungen sowie organisch bedingte Depression eingeschlossen sind.

2. Das Design der Studie machte es erforderlich, dass die beteiligten Ärzte mit den Studienzielen vertraut waren und somit ihre Aufmerksamkeit mehr als im normalen Praxisalltag auf depressive Symptome gelenkt haben könnten. Zudem ist durch die wiederholte Anwendung der Beurteilungskriterien von einem Lerneffekt auszugehen. 3. Auch konnte aufgrund des Designs nicht sichergestellt werden, dass die von den Ärzten indizierten Interventionen auch tatsächlich so durchgeführt wurden.

Diese Einschränkungen müssen gegen die Möglichkeiten abgewogen werden, die sich ergeben, wenn eine große Anzahl an Primärärzten und ihrer Patienten bundesweit systematisch untersucht werden kann.

\section{Prävalenz}

Die Validität der gefundenen Prävalenzrate von 11,3\% wird indirekt durch 3 Befunde gestützt:

1. Die Ergebnisse bewegen sich im Bereich derer, die in anderen Studien zu Depressionen in der Allgemeinarztpraxis gefunden wurden $[5,12,23]$, auch wenn dort z. T. andere Erhebungsmethoden bzw. klinische Interviews (allerdings mit identischen Kriterien) eingesetzt worden waren. Damit erscheint der Befund, dass etwa jeder 10. Patient betroffen ist, robust zu sein und unterstreicht, dass depressive Syndrome im primärärztlichen Setting eine überaus häufige Erkrankung darstellen.

2. Die Konsistenz der Ergebnisse ist auch über die teilnehmenden Ärzte hinweg, unabhängig von Art, Größe und regionaler Verteilung der Arztpraxen ausgesprochen hoch (range: 10,6$12,0 \%)$.

3. $95 \%$ der DSM und $87 \%$ der ICD-Fälle wurden von den Ärzten auf einem globalen Maß zur klinischen Auffälligkeit (CGI) als zumindest grenzwertig erachtet (selbst wenn nicht in allen Fällen auch die Diagnose vergeben wurde).

Die Differenz zwischen DSM- und ICD-Prävalenz (4,2\% gegenüber 11,1\%) ist auffällig und wurde so in früheren Studien noch nicht beschrieben. Dass die theoretisch eigentlich geringe Unterschiedlichkeit in der Konzeption der Grenze zur klinische Diagnose (ICD: 2 von 3 Leitsymptomen plus 2 zusätzliche Depressionssymptome vs. DSM: insgesamt 5 Symptome) zu einer so deutlichen Prävalenzdifferenz führt, weist darauf hin, dass sich viele der Fälle, die sich in der Allgemeinarztpraxis vorstellen, in ihrem Bild zwischen diesen beiden Grenzen bewegen. Die große Häufigkeit der schwellennahen Depressionen scheint für die 
primärärztliche Praxis charakteristisch zu sein und stellt für die Ärzte eine besondere Herausforderung dar.

\section{Erkennensraten}

Die Erkennensraten unserer Studie von nahezu 75\% zumindest auf der Fallerkennungsebene liegen bedeutsam höher als in früheren Studien, in denen sie bei gleichen Konventionen lediglich um 50\% schwankten [10]. Die Sensitivität der ärztlichen Diagnose Depression darf als ermutigend betrachtet werden, liegt allerdings offensichtlich hoch zu Lasten häufiger falsch positiver Befunde. Sie scheint anzudeuten, dass die verstärkte Aufmerksamkeit, die dem Problem depressiver Syndrome in der Hausarztpraxis in den letzten 10 Jahren gewidmet wurde, zu der verbesserten Entdeckung beigetragen haben. Problematisch muss jedoch die noch hohe Rate falsch-positiver Depressionsdiagnosen gesehen werden, die wir kürzlich ausführlich erörtert haben [8].

Interessant ist weiterhin die Frage, was die Güte der ärztlichen Diagnostik beeinflusst. Die Rolle von Patienten-, Krankheits- und Arztvariablen wurde bereits andernorts beschrieben $[26,27]$. Danach werden jüngere Patienten mit einer Depression, Männer sowie Patienten mit einem niedrigen Depressionswert vom Arzt signifikant schlechter erkannt als ältere Patienten, Frauen und schwere Depressionen. Den stärksten Einfluss auf die Erkennensrate hat jedoch der initiale, spontan vom Patient dem Arzt gegenüber geschilderte Konsultationsanlass in Kombination mit Depressionsschwere und bekannten früheren Behandlungen. Wann immer ein Patient psychische oder seelische Probleme schildert, steigt die Wahrscheinlichkeit des Erkennens einer Depression deutlich an. Ein früher postulierter Prädiktor für die Erkennensleistung, die Zahl der Arbeitsunfähigkeitstage in den letzten 4 Wochen [5], konnte in der vorliegenden Studie nicht bestätigt werden.

Arzt- und Praxisvariablen erwiesen sich überraschenderweise kaum als mit der Erkennensleistung assoziiert. Es bestand lediglich eine von der Größe her nicht beeindruckende Tendenz dahingehend, dass in erster Linie diejenigen, die sich aktuell und kontinuierlich mit der Problematik psychischer Störungen auseinandersetzen, bessere Erkennensqualitäten aufweisen, nicht jedoch Ärzte, die bereits vor Jahren eine einschlägige Weiter- oder Zusatzqualifikation erworben haben. Ärzte, die angeben, häufig selbst behandeln zu müssen, weil keine entsprechenden Spezialisten in der Nähe vorhanden sind, Ärzte, die Depressionspatienten ,ungern“ behandeln, und Ärzte, die empfinden, dass depressive Patienten einen zu hohen Zeitaufwand erfordern, erkennen Depressionspatienten signifikant schlechter als ihre Kollegen, die keine entsprechenden Einstellungen angeben.

Eine eingehendere Untersuchung ergab, dass weder Merkmale der Verteilung bei den Depressionswerten noch Korrelate zu Erkennensraten und Behandlungsarten nahe legen, ob die ICD- oder die DSM-Definition angemessener ist; Erkennensraten und Behandlungen nahmen (bereits unterhalb der ICD-Grenze) einfach stetig mit steigender Symptomzahl zu [27].Auch die hohe Zahl der Patienten, die vom Arzt die Diagnose erhalten, obwohl sie sich laut DSQ-Wert selbst noch unter dem ICD-Grenzwert befinden, kann als Bestätigung früherer Studien betrachtet werden, in denen ein beträchtlicher Anteil an subklinischen Depressionen berichtet wurde. Derartige Bilder wurden und werden mit einer Vielzahl von Begriffen und Definitionen belegt, z.B. kurze wiederkehrende Depression, minore Depression, subsyndromale Depression oder Störung mit Angst-Depression gemischt [14]. Da in der vorliegenden Studie keine dieser Definitionen benutzt wurde, ist es nicht möglich zu entscheiden, welche der folgenden Aspekte für die ,falschen Positiven“ verantwortlich ist: 1. Die Ärzte könnten in der nicht alltäglichen Situation am Studientag systematisch auch bei geringer Symptomzahl oder bei anderen psychischen Störungen eine Depression diagnostiziert haben (vielleicht, weil sie dies für erwünscht hielten); 
2. die Ärzte könnten Entscheidungsregeln benutzen, die in unseren nosologisch orientierten Diagnosesystemen nicht vertreten sind;

3. die Ärzte könnten aufgrund des fluktuierenden Verlaufs von Depressionen und ihrer eingehenderen Kenntnis der Patienten und deren Krankheitsgeschichte schneller diagnostiziert haben, z. B. wenn sie den Eindruck haben, dass sich erste Zeichen einer neuen Phase bei einem Patienten ankündigen, der in der Vergangenheit bereits depressive Episoden aufwies;

4. die Ärzte könnten grundsätzlich nicht dazu in der Lage sein, korrekte

Depressionsdiagnosen abzuleiten.

Oftmals benutzen Ärzte den Begriff „Depression“ zur Bezeichnung von nicht näher spezifizierten Beschwerde-, Belastungs- und Klagesyndromen. Hierfür sprechen die „verbatim“ geäußerten Patientenbeschreibungen im Arztbogen. Die Klärung dieses Punktes hat eine kritische Bedeutung für zukünftige Studien, die derzeitig geplant werden. Eine eingehendere Analyse zum Phänomen der ,falschen Positiven“ findet sich unter [8].

\section{Behandlung}

Unter der Annahme, dass „klassische“ und „moderne“ Antidepressiva, Beratung/ Krisenintervention, psychologische Behandlung und Überweisung zum Spezialisten (Psychiater, Nervenarzt, Psychotherapeut, psychiatrische Klinik) adäquate Behandlungsstrategien für den Hausarzt bei erkannter Depression darstellen, ist das Bild von Erkennung und Behandlung der Ärzte in unserer Studie bedeutend vorteilhafter als in beinahe allen früheren Studien. Hierbei muss berücksichtigt werden, dass in dieser Stichprobe „Hochverschreiber“- Praxen möglicherweise etwas überrepräsentiert sein könnten. Seltener waren neben dem vergleichsweise niedrigen Anteil von Depressionen ohne jegliche Intervention (DSM: 4,5\%; ICD: 11\%) pflanzliche Verschreibungen (18,9\% bzw. 22,6\%), Verschreibung von Sedativa/Hypnotika (8,9\% bzw. 6,6\%) und Neuroleptika (4,8\% vs. 3,2\%) sowie Beratung ohne weitere Maßnahmen (11,5\% vs. 18,8\%). Allerdings konnte die adäquate Umsetzung der angegebenen Interventionen durch die Ärzte sowie die Effektivität der Maßnahmen in dieser (querschnittlichen) Studie nicht untersucht werden - auch eine evidenzbasierte Intervention kann bei zu langer oder zu kurzer Dauer, frühzeitigem Therapieabbruch oder unangepasster Dosis wirkungslos bleiben [19].

Der mit 11,3\% hohe Anteil von Patienten, die die ärztliche Diagnose erhielten, ohne die Forschungskriterien zu erfüllen, wurde fast identisch behandelt. Auch wenn die größere Indikationsbreite von Antidepressiva berücksichtigt wird (z.B. bei somatoformen und Angststörungen), ist die hohe Verschreibungsquote bei Fällen ohne bzw. mit wenigen Symptomen bzw. unterschwelliger Depressionssymptomatik auffällig.

In diesem Zusammenhang lassen sich eine Reihe kritischer Fragen aufwerfen: Sind die kurzund langfristigen Effekte antidepressiver medikamentöser Behandlung bei solchen unterschwellig depressiven Fällen ähnlich günstig denen bei schwereren Depressionen, für die die Medikamente eigentlich entwickelt wurden? Sind die unterschwellig depressiven Fälle vielleicht anfälliger für Nebenwirkungen und brechen Behandlungen vorzeitig ab? Wenn sich dadurch das Effektivitätsprofil des Medikaments verschlechtert, wie wirkt sich das langfristig auf die Bereitschaft des Arztes aus, (auch schwerere) Depressionen mit dem Medikament zu behandeln? Es handelt sich also um ein relevantes Problem, für das die Übertragung von Befunden aus dem psychiatrischen und klinischen Bereich problematisch erscheint und für das trotz seiner Bedeutung nach wie vor zu wenige wissenschaftliche Daten vorliegen. (Zentrale bzw. weiterführende Literatur s. [8, 19, 23, 25, 26].) 
Tabelle 1

Sozlodemographlsche Merkmale der Patientenstichprobe ( $N=20.421)$

\begin{tabular}{|c|c|c|c|c|c|c|}
\hline & \multicolumn{2}{|c|}{ Mànner } & \multicolumn{2}{|c|}{ Fraven } & \multicolumn{2}{|c|}{ Gesamt ${ }^{2}$} \\
\hline & $N$ & {$[9](95 \% \mathrm{Cl})$} & $N$ & [\%] $(95 \% \mathrm{Cl})$ & $N$ & {$[90](95 \% \mathrm{Cl})$} \\
\hline \multicolumn{7}{|l|}{ Alter [lahre] } \\
\hline $15-24$ & 598 & $7,8(7,0-8,6)$ & 916 & $7,9(7,3-8,6)$ & 1591 & $7,8(7,31-8,30)$ \\
\hline $25-44$ & 1995 & $26,0(248-27,2)$ & 3418 & $29,6(28,4-30,8)$ & 5693 & $27,9(26,9-28,9)$ \\
\hline $45-64$ & 3124 & $40,7[39,4-42,0)$ & 4101 & $35,5(34,4-36,6)$ & 7653 & $37,5(36,5-38,4)$ \\
\hline $65+$ & 1961 & $25,5(24,3-26,8)$ & 3114 & $27,0(25,8-28,2)$ & 5484 & $26,9(25,8-27,9)$ \\
\hline \multicolumn{7}{|l|}{ Famillenstand ( $N=18.961)$} \\
\hline Ledig & 1564 & $21,7(20,5-22,9)$ & 1954 & $18,3(17,4-19,2)$ & 3695 & $19,5(18,7-20,3)$ \\
\hline Verhefratet & 4961 & $68,7(67,3-70,0)$ & 6175 & $57,7(56,6-58,9)$ & 11.756 & $62,0(61,0-63,0)$ \\
\hline Geschleden/getrennt & 700 & $9,7(8,9-10,5)$ & 2570 & $24,0(23,1-25,0)$ & 3500 & $18,5(17,8-19,2)$ \\
\hline \multicolumn{7}{|c|}{ Beruflicher Status ( $N=19.257)$} \\
\hline Benufstatig & 3854 & $52,9(51,3-54,3)$ & 4705 & $43,2(42,0-44,5)$ & 8985 & $46,7(45,6-47,7)$ \\
\hline Hausirau & 12 & $0,2(0,0-0,3)$ & 2281 & $21,0(19,6-22,4)$ & 2463 & $12,8(12,0-13,7)$ \\
\hline Arbettslos & 679 & $9,3(8,5-10,2)$ & 802 & $7, A(6,7-8,1)$ & 1571 & $8,2(7,5-8,8)$ \\
\hline Berentet/pensionilert & 2748 & $37,7(36,2-39,2)$ & 3096 & $28,5(27,1-29,8)$ & 6238 & $32,4[31,2-33,6)$ \\
\hline \multicolumn{7}{|c|}{ Anlass des Aratbesuches ${ }^{b}(N=14.777)$} \\
\hline Psychische Probleme & 493 & $8,8(7,9-9,7)$ & 1089 & $13,1(12,2-14,1)$ & 1686 & $11,4(10,7-12,2)$ \\
\hline Schlafprobleme & 1690 & $30,0[28,5-31,6]$ & 2999 & $36,1(34,9-37,4)$ & 5004 & $33,9(32,8-35,0)$ \\
\hline Schmerzen & 2499 & $44,4(42,8-46,0)$ & 3901 & $47,0(45,6-48,4)$ & 6797 & $46,0(44,8-47,2)$ \\
\hline Körperliche Beschwerden & 3997 & $71,0(69,5-72,5)$ & 5407 & $65,1(63,8-66,4)$ & 9953 & $67,4(66,3-68,4)$ \\
\hline Anderer Anlass & 601 & $10,9(10,0-11,8)$ & 923 & $11,1(10,3-11,9)$ & 1644 & $11,1(10,5-11,8)$ \\
\hline
\end{tabular}

a $\mathrm{N}$ fir Mönner ( $\mathrm{N}=7678)$ und Froven (N=11.549) addleren sich nicht zu 20.421, da von 1194 Potlenten de Angabe zum Geschlecht fehit;

"Mehrfochangaben möglich

Tabelle 2

DSQ-Punktprävalenzen In der hausärztlichen Praxis (nach Geschlecht und Alter für depressive Störungen nach DSM-IV und ICD-10; $N=20.304^{\mathrm{a}}$ )

\begin{tabular}{|c|c|c|c|c|c|c|c|}
\hline & \multirow{2}{*}{$\begin{array}{l}\text { Alter } \\
\text { [Jahre] }\end{array}$} & \multicolumn{2}{|c|}{ Männer } & \multicolumn{2}{|c|}{ Fraven } & \multicolumn{2}{|c|}{ Gesamt } \\
\hline & & $N$ & {$[\%](95 \% \mathrm{Cl})$} & $N$ & {$[96](95 \% \mathrm{Cl})$} & $N$ & {$[\%](95 \% \mathrm{Cl})$} \\
\hline Keine depressive & & 6975 & $91,3(90,4-95$ & 9989 & $87,0^{4}(86,1-87,6)$ & 18.004 & $88,7\{88,0-89,4\}$ \\
\hline \multirow[t]{4}{*}{ Störung (DSQ<8) } & $15-24$ & 543 & $91,7(89,0-93$ & 785 & $86,2^{*}(83,8-88,2)$ & 1395 & $88,4(86,7-89,9)$ \\
\hline & $25-44$ & 1837 & $92,3(90,9-93$ & 3015 & $88,5^{+}(87,3-89,6)$ & 5105 & $90,0(89,1-90,8)$ \\
\hline & $45-64$ & 2806 & $90,1(88,7-9)$ & 3548 & $86,7^{*}(85,5-88,1)$ & 6711 & $88,0(86,9-89,0)$ \\
\hline & $65+$ & 1789 & $92,0\{90,6-9\}$ & 2641 & $85,9^{*}(84,3-87,3)$ & 633 & $88,3(87,2-89,4)$ \\
\hline Irgendelne depresstve & & 667 & $8,7(7,9-9,6)$ & 1491 & $13,0^{4}(12,2-13,9)$ & 2300 & $11,3(10,6-120)$ \\
\hline \multirow{4}{*}{ Stărung (CD oder DSM) } & $15-24$ & 49 & $8,3(6,2-11,0)$ & 126 & $13,8^{4}(11,8-16,2)$ & 183 & $11,6(10,1-13,3)$ \\
\hline & $25-44$ & 153 & $7,7(6,5-9,1)$ & 393 & $11,5^{4}(10,4-12,7)$ & 569 & $10,0(9,2-10,9)$ \\
\hline & $45-64$ & 310 & $10,0(8,7-89,1)$ & 537 & $13,2^{4}(11,9-14,5)$ & 915 & $12,0(11,0-13,1)$ \\
\hline & $65+$ & 155 & $8,0(6,8-9,4)$ & 435 & $14,1^{*}(12,7-15,7)$ & 633 & $11,7(10,6-12,8)$ \\
\hline \multirow[t]{5}{*}{ Davon DSM-N } & & 234 & $3,1(2,6-3,6)$ & 560 & $4,9^{4}(4,3-5,5)$ & 849 & $4,2(3,8-4,7)$ \\
\hline & $15-24$ & 15 & $2,5(1,5-4,2)$ & 41 & $4,5(3,3-6,1)$ & 58 & $3,7(2,8-4,7)$ \\
\hline & $25-44$ & 54 & $2,7(2,0-3,6)$ & 148 & $4,3(3,6-5,2)$ & 213 & $3,8(3,2-4,4)$ \\
\hline & $45-64$ & 111 & $3,6(2,9-4,4)$ & 218 & $5,3^{*}(4,5-6,3)$ & 358 & $4,7(4,1-5,4)$ \\
\hline & $65+$ & 54 & $2,8(2,1-3,7)$ & 153 & $5,0^{4}(4,1-6,1)$ & 220 & $4,1(3,4-4,8)$ \\
\hline \multirow[t]{5}{*}{ Nur KCD-10 } & & 433 & $5,7(5,1-6,3)$ & 931 & $8,1 * 07,5-8,7)$ & 1451 & $7,2(6,7-7,6)$ \\
\hline & $15-24$ & 34 & $5,7(4,1-8,0)$ & 85 & $9,3(7,6-11,4)$ & 125 & $7,9(6,6-9,4)$ \\
\hline & $25-44$ & 99 & $5,0(4,0-6,1)$ & 245 & $7,2^{*}(6,3-8,2)$ & 356 & $6,3(5,6-7,0)$ \\
\hline & $45-64$ & 199 & $6,4(5,4-7,5)$ & 319 & $7,8^{4}(6,9-8,8)$ & 557 & $7,3(6,6-8,1)$ \\
\hline & $65+$ & 101 & $5,2(4,2-6,3)$ & 282 & $9,2^{*}(8,1-10,4)$ & 413 & $7,6(6,8-8,5)$ \\
\hline
\end{tabular}

a 117 Falle wurden wegen fehiender OSQ-Werte won der Analyse ausgeschlossen, "slgnifikanter Geschlechtsunterschled ( $p<$ 05) 


\begin{tabular}{|c|c|c|c|c|c|c|c|c|c|c|}
\hline \multirow{3}{*}{$\begin{array}{l}\text { Arztliche } \\
\text { Elnschatrang }\end{array}$} & \multirow{2}{*}{\multicolumn{2}{|c|}{ Gesamt }} & \multicolumn{8}{|c|}{ Dlagnosestatus (DSQ) } \\
\hline & & & \multicolumn{2}{|c|}{ DSM-AV } & \multicolumn{2}{|c|}{ Nur ICD-10 } & \multicolumn{2}{|c|}{ DSM and ICD gesamt } & \multicolumn{2}{|c|}{ Weder DSM noch ICD } \\
\hline & N & {$[\%](95 \% \mathrm{Cl})$} & $N$ & {$[9](9596 \mathrm{Cd})$} & $N$ & [96] $(95 \% C D)$ & $N$ & {$[96](95 \% \sigma)$} & $N$ & {$[9](95 \% \mathrm{C}]$} \\
\hline $\begin{array}{l}\text { Definitivkeine } \\
\text { Depression }\end{array}$ & 9096 & $\begin{array}{l}47,4 \\
(45,9-48,9)\end{array}$ & 33 & $\begin{array}{l}4,1 \\
(2,7-6,1)\end{array}$ & 138 & $\begin{array}{l}10,0 \\
(8,3-11,9)\end{array}$ & 171 & $\begin{array}{l}7,8 \\
(6,5-9,3)\end{array}$ & 8897 & $\begin{array}{l}52,6 \\
(51,0-54,2)\end{array}$ \\
\hline Unklar & 1481 & $\begin{array}{l}7,7 \\
(7,0-8,5)\end{array}$ & 32 & $\begin{array}{l}4,0 \\
{[2,8-5,6)}\end{array}$ & 123 & $\begin{array}{l}8,9 \\
(7,4-10,6)\end{array}$ & 155 & $\begin{array}{l}7,1 \\
(5,9-8,4)\end{array}$ & 1313 & $\begin{array}{l}7,8 \\
(7,0-8,6)\end{array}$ \\
\hline $\begin{array}{l}\text { Unterschwellige } \\
\text { Depression }\end{array}$ & 5327 & $\begin{array}{l}27,8 \\
(26,7-28,8)\end{array}$ & 139 & $\begin{array}{l}17,2 \\
(14,4-20,3)\end{array}$ & 437 & $\begin{array}{l}31,5 \\
(28,8-34,3)\end{array}$ & 576 & $\begin{array}{l}26,2 \\
(24,1-28,5)\end{array}$ & 4728 & $\begin{array}{l}28,0 \\
(26,8-29,2)\end{array}$ \\
\hline $\begin{array}{l}\text { Sicher und } \\
\text { voll ausgeprigt }\end{array}$ & 3290 & $\begin{array}{l}17,1 \\
(16,2-18,2)\end{array}$ & 606 & $\begin{array}{l}74,8 \\
(71,3-78,0)\end{array}$ & 689 & $\begin{array}{l}49,7 \\
(46,5-52,8)\end{array}$ & 1295 & $\begin{array}{l}59,0 \\
(56,3-61,5)\end{array}$ & 1971 & $\begin{array}{l}11,7 \\
(10,8-12,6)\end{array}$ \\
\hline Summe & 19.194 & 100 & 810 & 100 & 1387 & 100 & 2197 & 100 & 16.909 & 100 \\
\hline
\end{tabular}

\begin{tabular}{|c|c|c|c|c|c|c|}
\hline \multirow{2}{*}{$\begin{array}{l}\text { Intervention bel den } \\
\text { Arztdlagnosen }(N=8181)^{p}\end{array}$} & \multicolumn{2}{|c|}{ Falle nach DSM-IV } & \multicolumn{2}{|c|}{ Fallenach ICD-10 } & \multicolumn{2}{|c|}{ Falsche Positive ${ }^{2}$} \\
\hline & $N$ & [\%] $(95 \%$ C) & N & [\%] $(95 \% \mathrm{Cl})$ & $N$ & [\%] $(95 \% \mathrm{Cl})$ \\
\hline Nicht erkannt & 65 & $8,0(6,0-10,6)$ & 261 & $18,8(16,4-21,4)$ & - & - \\
\hline Kelne Intervention & 32 & $4,5(3,1-6,4)$ & 120 & $11,0(9,0-13,4)$ & 130 & $5,9(5,0-7,0)$ \\
\hline Oberwelsung an Sperlalisten & 115 & $16,2(13,3-19,5)$ & 110 & $10,1(8,2-12,4)$ & 220 & $11,7(9,9-13,7)$ \\
\hline Nur Beratung & 82 & $11,5(9,3-14,2)$ & 205 & $18,8(16,3-21,7)$ & 271 & $14,4(13,3-16,7)$ \\
\hline Psychotheraple (ohne Medlikamente) & 59 & $8,3(6,4-10,6)$ & 66 & $6,1(4,7-7,9)$ & 102 & $5,4(4,3-6,7)$ \\
\hline Irgendeine Medikation & 517 & $72,7(69,0-76,2)$ & 661 & $60,8(57,2-64,2)$ & 1,316 & $69,8(66,8-72,7)$ \\
\hline Hypnotika/Sedativa & 63 & $8,9(6,5-11,9)$ & 72 & $6,6(5,0-8,7)$ & 142 & $7,5(6,0-9,4)$ \\
\hline Pflandiche Prăparate & 134 & $18,9(15,9-22,3)$ & 246 & $22,6(19,9-25,5)$ & 424 & $22,5(19,9-25,3)$ \\
\hline Neurcleptika & 34 & $4.8(3,4-6,7)$ & 35 & $3,2(2,2-4,6)$ & 88 & $4,7(3,5-6,2)$ \\
\hline Massische* Antidepressiva (TCA) & 197 & $27,7(24,3-31,4)$ & 176 & $16,2(13,8-18,9)$ & 449 & $23,8(21,4-26,4)$ \\
\hline $\begin{array}{l}\text { Moderne" Antidepressiva } \\
\text { (SSRI,SNRL, NaSSA etc.) }\end{array}$ & 267 & $37,6(33,1-42,29)$ & 306 & $28,1(25,0-31,4)$ & 593 & $31,5(28,4-34,7)$ \\
\hline Andere & 22 & $3,1(1,9-4,8)$ & 25 & $2,3(1,5-3,5)$ & 55 & $2,9(2,0-4,1)$ \\
\hline
\end{tabular}

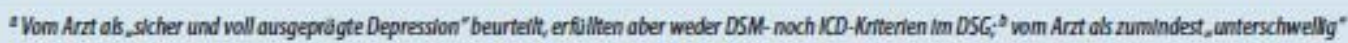
beurtellt; Mehrfochangaben möglıch; mirsings gegenuber Tabelle 3 wegen z T. fehlender Angaben zur Intervention

Die Durchführung dieser Studie wurde mit einem „unrestricted educational grant““ der Firma SmithKline Beecham (Deutschland) unterstützt.

\section{Literatur}

1. Barsky AJ, Borus JB (1995) Somatization and medicalization in the era of managed care. J Am Med Assoc 274:1931-1934

2. Eisenberg L (1992) Treating depression and anxiety in primary care: closing the gap between knowledge and practice. N Engl JMed 326:1080-1083

3. Goldberg D (1979) Detection and assessment of emotional disorders in primary care setting. Int J Mental Health 8:30-48

4. Goldberg D (1995) Epidemiology of mental disorders in primary care settings. Epidemiol Rev 7: 182-190

5. Goldman LS, Nielsen NH, Champion HC (1999). Awareness, diagnosis, and treatment of depression. J Gen Internal Med 14:569-580

6. Guy W, Bonato R (1970) CGI: Clinical Global Impressions: Manual for the ECDEU Assessment Battery 2, revised edn. National Institute of Mental Health, Baltimore 
7. Hirschfeld R, Keller MB, Panico S et al. (1997) The National Depressive and Manic--Depressive Association Consensus Statement on the Undertreatment of Depression. J Am Med Assoc 277:333340

8. Höfler M, Wittchen HU (2000) Why do primary care dorctors diagnose depression when diagnostic criteria are not met? Int J Meth Psychiatr Res 9:110-120

9. Kassenärztliche Bundesvereinigung (1999) Grunddaten zur vertragsärztlichen Versorgung in der Bundesrepublik Deutschland. Dtsch Ärzte-Verlag, Köln

10. Lecrubier Y (1998) Is depression under-recognised and undertreated? Int Clin Psychopharmacol 13 [Suppl 5]:3-6

11. Lepine JP, Gastpar M, Mendlewicz J, Tylee A (1997) Depression in the community: The first panEuropean Study DEPRES. Int Clin Psychopharmacol 12(1): 19-29.

12. Linden M, Maier W, Achenberger M, Herr R (1996) Psychiatrische Erkrankungen und ihre Behandlung in Allgemeinarztpraxen in Deutschland: Ergebnisse einer Studie der WHO. Nervenarzt 67(3):205-215

13. Ormel J, Koeter MWJ, van den Brink W, van de Willige G (1991) Recognition, management, and course of anxiety and depression in general practice. Arch Gen Psychiatry 48:700-706

14. Pincus HA, Davis WW, McQueen LE (1999) „Subthreshold“ mental disorders. A review and synthesis of studies on minor depression and other „brand names“.Br J Psychiatry 174:288-296 15. Regier DA, Hirschfeld RMA, Goodwin FK, Burke JD, Lazar JB, Judd LL (1988) The NIMH Depression Awareness. Recognition and treatment program: structure, aim, and scientific basis. Am J Psychiatry 145:1351-1357

16. Schulberg HC, Block MR, Madonia MJ et al. (1996) Treating major depression in primary care practice. Arch Gen Psychiatry 53:913-919

17. Simon GE, Goldberg D, Tiemens BG, Üstün TB (1999) Outcomes of recognized and unrecognized depression in an International Primary Care Study. Gen Hosp Psychiatry 21:97-105

18. Simon GE, von Korff M (1995) Recognition, management, and outcomes of depression in primary care. Arch Fam Med 4:99-105

19. Simon GE, Ludman E (2000) Lessons from recent research on depression in primary care.

Epidemiol Psichiatria Soc 9:145-151

20. Tiemens BG, Ormel J, Simon GE (1996) Occurrence, recognition, and outcome of psychological disorders in primary care. Am J Psychiatry 153:636-644

21. Tylee A, Freeling P, Kerry S (1993) Why do general practioners recognize major depression in one woman patient yet miss it in another? Br J Gen Pract 43:327-330

22. Tylee A, Gastpar M, Lepine JP, Mendlewicz J (1999) DEPRES II: A patient survey of the symptoms, disabilit y and current management of depression in the community. Int Clin Psychopharmacol 14(3): 139-151

23. Üstün TB, Sartorius N (1995) Mental illness in General Health Care across the world. An international study. John Wiley \& Sons, New York

24. von Korff M, Shapiro S, Burke JD et al. (1987) Anxiety and depression in a primary care clinic: comparison of Diagnostic Interview Schedule, General Health Questionnaire and practitioner Assessments. Arch Gen Psychiatry 44:152-156

25. Wittchen HU, Höfler M (in press) Psychometric properties of the DSQ questionnaire. Int JMeth Psychiatr Res

26. Wittchen HU, Höfler M, Meister W (2000) Depressionen in der Allgemeinpraxis. Die bundesweite Depressionsstudie. Mit einem Geleitwort von Prof. Dr. med. S. Kasper. Schattauer, Stutt gart

27. Wittchen HU, Höfler M, Meister W (in press) Prevalence and recognition of depressive syndromes in German primary care settings - poorly recognized and treated? Psychol Med 28. Wittchen HU, Pfister H (eds) (1997) DIA-X-Interviews: Manual für Screening-Verfahren und Interview; Interviewheft Längsschnittuntersuchung (DIA-X-Lifetime); Ergänzungsheft (DIA-XLifetime); Interviewheft Querschnittuntersuchung (DIA-X-12 Monate); Ergänzungsheft (DIA-X-12 Monate); PC-Programm zur Durchführung des Interviews (Längs- und Querschnittuntersuchung); Auswertungsprogramm. Swets, Zeitlinger, Frankfurt 